\title{
Non-crosslinked porcine-derived acellular dermal matrix for the management of complex ventral abdominal wall hernias: a report of 45 cases
}

\author{
O. Guerra • M. M. Maclin
}

Received: 13 August 2012/Accepted: 28 July 2013/Published online: 10 August 2013

(C) The Author(s) 2013. This article is published with open access at Springerlink.com

\begin{abstract}
Purpose Ventral abdominal wall hernias are common and repair is frequently associated with complications and recurrence. Although non-crosslinked intact porcinederived acellular dermal matrix (PADM) has been used successfully in the repair of complex ventral hernias, there is currently no consensus regarding the type of mesh and surgical techniques to use in these patients. This report provides added support for PADM use in complex ventral hernias.

Methods In a consecutive series of adult patients (2008-2011), complex ventral abdominal wall hernias (primary and incisional) were repaired with PADM by a single surgeon. Patient comorbidities, repair procedures, and postoperative recovery, recurrence, and complications were noted.

Results Forty-four patients (mean age, 57.5 years) underwent 45 single-stage ventral abdominal wall hernia repairs ( 3 primary; 42 incisional). Previously placed synthetic mesh was removed in 17 cases. In 40 cases, primary fascial closure was achieved; in 5 cases, PADM was used as a bridge. Vacuum-assisted closure (VAC) was used for 38/45 cases: 19 closed incisions, 16 cases using the "French fry" technique, and 3 cases with open incisions.
\end{abstract}

Electronic supplementary material The online version of this article (doi:10.1007/s10029-013-1148-x) contains supplementary material, which is available to authorized users.

O. Guerra $(\bowtie)$

Suburban Surgical Associates, 555 N. New Ballas Rd.,

Ste. 265, St. Louis, MO 63141, USA

e-mail: omarg@ssainc.net

M. M. Maclin

Parkcrest Plastic Surgery, St. Louis, MO, USA
Mean hospital stay was 8.2 days (range, 3-32) and mean follow-up was 17 months (range, 1-48). There were 4 $(8.9 \%)$ hernia recurrences, 3 requiring additional repair and 1 requiring PADM explantation. There were $3(6.7 \%)$ skin dehiscences, $4(8.9 \%)$ deep wound infections requiring drainage, and $5(11.1 \%)$ seromas (4 self-limited, 1 requiring drainage).

Conclusions Non-crosslinked intact PADM yielded favorable early outcomes when used to repair complex ventral abdominal wall hernias in high-risk patients.

Keywords Ventral abdominal wall hernias .

Non-crosslinked intact porcine-derived acellular dermal matrix $\cdot$ Vacuum-assisted closure $\cdot$ Synthetic mesh $\cdot$ Biologic acellular dermal matrix $\cdot$ Strattice

\section{Introduction}

The ventral abdominal wall is second to the inguinal region for the most common types of abdominal hernia and may lead to life-threatening complications [1]. Ventral incisional hernias are typically seen as postoperative complications of abdominal surgery, with a reported incidence of 11 to $19 \%$ in patients undergoing laparotomy $[2,3]$. Risk factors for incisional hernia include obesity, diabetes, emergency surgery, postoperative wound dehiscence, smoking, and postoperative wound infection [4-6].

To help surgeons stratify patient risk of developing postoperative complications and promote improved patient selection for different surgical repair approaches, the Ventral Hernia Working Group (VHWG) proposed a fourlevel surgical-site occurrence risk grading system (Fig. 1) [7]. For healthy patients without significant comorbidities (Grade 1), prosthetic mesh is recommended (vs suture 
Fig. 1 Ventral Hernia Working Group (VHWG) hernia grading system. Reproduced from Breuing et al. [7] (C2010, with permission from Elsevier

\begin{tabular}{|c|c|c|c|}
\hline Grade 1 & Grade 2 & Grade 3 & Grade 4 \\
\hline $\begin{array}{l}\text { Low Risk } \\
\text { - Low risk of } \\
\text { complications } \\
\text { - No history of } \\
\text { wound infection }\end{array}$ & $\begin{array}{l}\text { Comorbid } \\
\text { - Smoker } \\
\text { - Obese } \\
\text { - Diabetic } \\
\text { - Immunosuppressed } \\
\text { - COPD }\end{array}$ & $\begin{array}{l}\text { Contaminated } \\
\text { - Previous wound } \\
\text { infection } \\
\text { - Stoma present } \\
\text { - Violation of GI tract }\end{array}$ & $\begin{array}{l}\text { Infected } \\
\text { - Infected mesh } \\
\text { - Septic } \\
\text { dehiscence }\end{array}$ \\
\hline
\end{tabular}

repair alone) for reducing recurrence [7, 8]. Use of a biologic matrix rather than synthetic mesh should be considered in patients with comorbidities (Grade 2) because of their increased risk of surgical-site occurrences [7]. For patients with a high risk of surgical-site occurrences due to contamination (Grade 3) or infection (Grade 4), biologic matrix repair is preferable to synthetic mesh repair for reducing recurrence [7, 9], though prospective, well-controlled trials comparing these mesh types are lacking.

Although synthetic mesh continues to be used in abdominal wall hernia repair, it is associated with postoperative complications including intestinal obstruction, fistula formation, extrusion, and infection [7]. Biologic matrices (such as ADM) have become more commonly used as effective alternatives to synthetic mesh in the setting of ventral herniorrhaphy. A non-crosslinked intact porcine-derived ADM (PADM; Strattice ${ }^{\mathrm{TM}}$ Reconstructive Tissue Matrix, LifeCell Corp., Branchburg, NJ, USA) is designed to perform as a surgical mesh for soft-tissue repair while serving as a scaffold for the rapid ingrowth of host cells, collagen, and blood vessels [10].

The objective of the current retrospective cohort study is to describe and evaluate repair procedures, postoperative complications, and short-term outcomes with PADM in a series of patients with complex primary and incisional ventral hernias. For the purposes of this study, a VHWG Grade 2, 3, or 4 risk classification connoted complex ventral hernia.

\section{Methods}

\section{Patients}

This was an Institutional Review Board-approved retrospective cohort study of all consecutive adult patients undergoing single-stage complex ventral herniorrhaphy (primary and incisional) with PADM by the senior author (O.G.) between 2008 and 2011. Details on patient demographics and comorbidities, history related to the hernias, and hernia characteristics were noted.

\section{Surgical repair procedures}

Surgical repair procedures were largely determined by the individual patient's condition and anatomic findings. Components separation was performed when primary fascial closure could not otherwise be achieved. Muscle and fascia repair was performed with \#1 Prolene ${ }^{\circledR}$ or polydioxanone (PDS; Ethicon, Inc., Somerville, NJ, USA) sutures. PADM was preferably used in the intraperitoneal or retrorectus position to reinforce the repairs, and was only used as a bridge when fascial closure could not be achieved. PADM was secured into position with transfascial \#1 Prolene or PDS sutures. Blake drains (size 19 French) were placed in most cases and secured to bulb suction. Wound closure, when performed, was done with sutures of appropriate type and size. Vacuum-assisted closure (VAC) was applied to reduce wound tension and promote healing. In some cases involving a "French fry" technique, strips of white foam $2 \mathrm{~cm}$ in width were inserted into portals of the incision (Fig. 2), after which a wound VAC was placed over the entire incision using black foam.

\section{Postoperative management}

Surgical drains were continued postoperatively until output was $<30 \mathrm{cc} /$ day for 2 consecutive days. Antibiotics were continued as needed for patients with infection or gross contamination. Pain management included epidural catheter or patient-controlled analgesia and then oral opioids.

\section{Postoperative assessments}

All patients were scheduled for follow-up office visits at 2 and 6 weeks and then yearly to address concerns, adverse events, or complications. Patients were monitored for any recurrences based on clinical examination in the office, by 

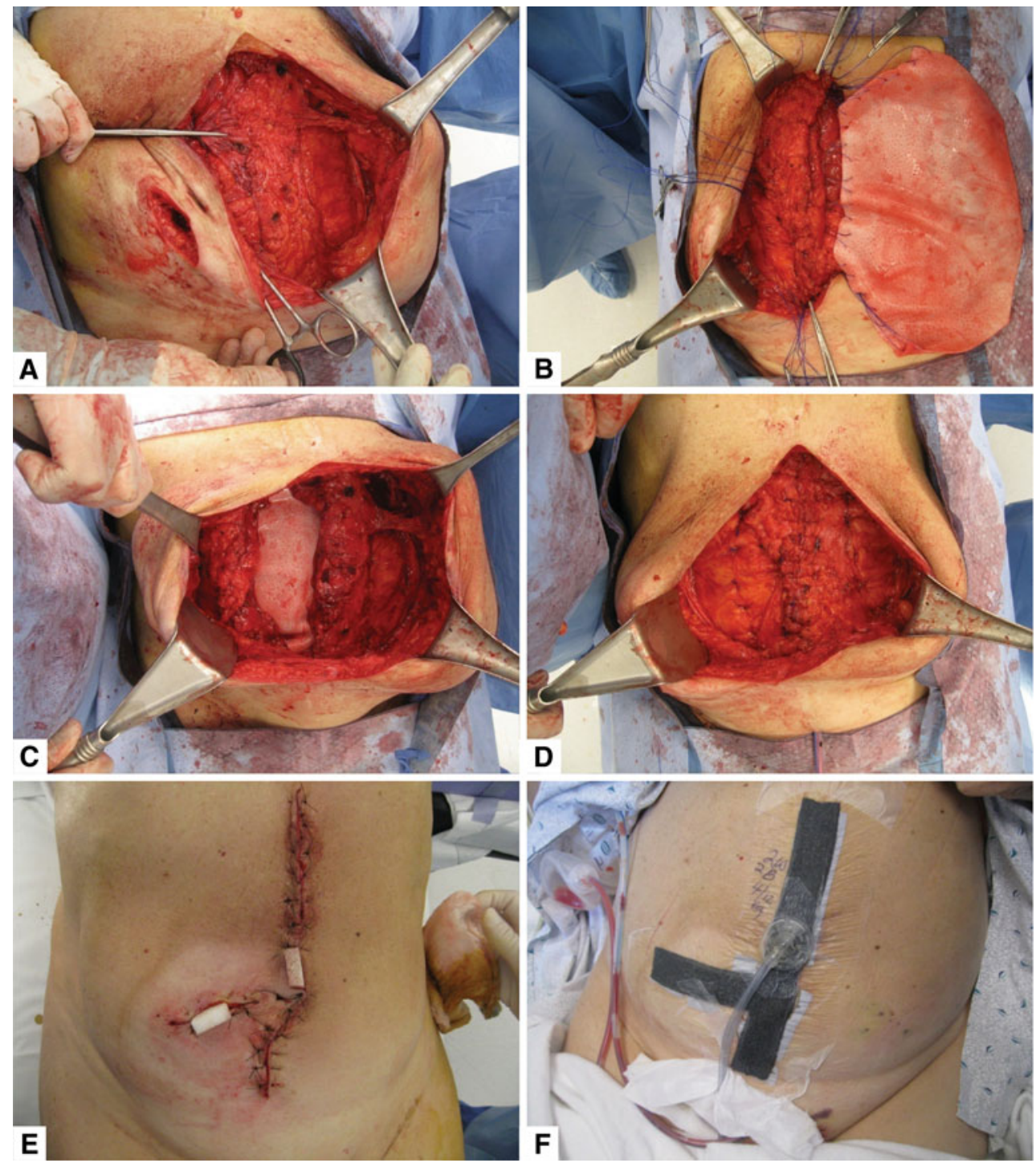

Fig. 2 Intraoperative photographs: a components separation; b PADM underlay using transfascial sutures; c completion of the underlay PADM placement; $\mathbf{d}$ completion of midline fascial closure;

the senior author. Complications and adverse events were documented.

\section{Results}

\section{Patients}

The demographic and clinical characteristics of patients are summarized in Table 1. Mean age was 57.5 years (range, 34-80) and mean body mass index (BMI) was $36.7 \mathrm{~kg} / \mathrm{m}^{2}$ (range, 21-65). The majority of procedures $(n=29)$ were performed in female patients. A total of 44 patients underwent 45 procedures ("cases"); 1 patient underwent 2 unrelated procedures and was considered as 2 separate

e "French fry" technique with 2 portals with white foam; f black foam placed over entire incision line; $P A D M$ non-crosslinked intact porcine-derived acellular dermal matrix

cases. She was initially treated for multisystem organ failure and fecal peritonitis (Grade 4 hernia); the defect was bridged with PADM. Two years later, she presented with a Grade 3 hernia, which was treated with components separation and PADM underlay.

\section{Preoperative clinical characteristics}

Three cases were primary hernias and 42 were incisional hernias (Table 1). Of the incisional hernias, 30 cases were following midline laparotomy, 8 were at midline and ostomy sites following laparotomy with ostomy takedown, 3 were following transverse rectus abdominis myocutaneous (TRAM) flap procedures, and 1 was at a right subcostal incision site. No cases qualified as VHWG Grade 1 (low 
Table 1 Patient demographics and clinical characteristics

\begin{tabular}{|c|c|}
\hline \multicolumn{2}{|l|}{ Age $(y)$} \\
\hline Mean & 57.5 \\
\hline Range & $34-80$ \\
\hline \multicolumn{2}{|l|}{ Sex, $n$} \\
\hline Male & 16 \\
\hline Female & $29^{\mathrm{a}}$ \\
\hline \multicolumn{2}{|l|}{ BMI, $\mathrm{kg} / \mathrm{m}^{2}$} \\
\hline Mean & 36.7 \\
\hline Range & $21-65$ \\
\hline \multicolumn{2}{|l|}{ Hernia type, $n$} \\
\hline Primary & 3 \\
\hline Incisional & 42 \\
\hline \multicolumn{2}{|l|}{ VHWG Grade, ${ }^{\mathrm{b}} n$} \\
\hline 1 & 0 \\
\hline 2 & 9 \\
\hline 3 & 25 \\
\hline 4 & 11 \\
\hline Mean & 3 \\
\hline \multicolumn{2}{|l|}{ Comorbidities, $n(\%)$} \\
\hline History of abdominal wall infection & $33(73)$ \\
\hline History of MRSA abdominal wall infection & $10(22)$ \\
\hline COPD/emphysema & $14(31)$ \\
\hline Malnutrition/low preoperative serum albumin & $12(27)$ \\
\hline Diabetes mellitus & $11(25)$ \\
\hline Hypertension & $11(25)$ \\
\hline Smoking & $9(20)$ \\
\hline Stoma present & $6(13)$ \\
\hline Renal failure & $2(4)$ \\
\hline Therapeutic use of corticosteroids & $2(4)$ \\
\hline Chronic panniculitis & $2(4)$ \\
\hline History of coronary artery bypass graft & $2(4)$ \\
\hline Breast cancer & $2(4)$ \\
\hline Congestive heart failure & $2(4)$ \\
\hline Atrial fibrillation & $2(4)$ \\
\hline Crohn's disease & $2(4)$ \\
\hline Thoracic aneurysm & $2(4)$ \\
\hline Other & $23(51)$ \\
\hline
\end{tabular}

a One patient presented 2 years after initial treatment. This patient had two separate procedures and was considered as two separate cases

b See Fig. 1 for explanation of VHWG grades

$B M I$ body mass index, $C O P D$ chronic obstructive pulmonary disease, MRSA methicillin-resistant $S$. aureus

risk), and the mean grade was 3. Comorbidities included history of abdominal wall infection $(n=33)$, obesity (BMI $>30 \mathrm{~kg} / \mathrm{m}^{2} ; n=27$ ), chronic obstructive pulmonary disease $(\mathrm{COPD}) /$ emphysema $(n=14)$, malnutrition $(n=12)$, hypertension $(n=11)$, diabetes mellitus $(n=11)$, and history of methicillin-resistant Staphylococcus aureus (MRSA) infection $(n=10)$. Six patients
Table 2 Surgical repair

\begin{tabular}{ll}
\hline Procedural information & Frequency, $n(\%)$ \\
\hline Synthetic mesh removed & $11(24)$ \\
Infected & $6(13)$ \\
Non-infected total & $17(38)$ \\
Total & \\
Successful fascial closure & $20(44)$ \\
Components separated & $20(44)$ \\
Components not separated & $40(89)$ \\
Total & \\
Failed fascial closure; PADM used as a bridge & $3(7)$ \\
Components separated & $2(4)$ \\
Components not separated & $5(11)$ \\
Total & \\
PADM placed & $39(87)$ \\
Intraperitoneal & $6(13)$ \\
Retrorectus & $45(100)$ \\
Total &
\end{tabular}

$P A D M$ non-crosslinked intact porcine-derived acellular dermal matrix

were obese and had malnutrition preoperatively. Four patients had a history of prior mesh removal (none infected), 3 of which were following TRAM flap procedures by another surgeon.

Operative techniques

Surgical details are summarized in Table 2. PADM was successfully placed in 45 cases using either intraperitoneal or retrorectus insertion; sheet sizes ranged from $6 \times 16 \mathrm{~cm}$ to $20 \times 30 \mathrm{~cm}$. In 40 cases, primary fascial closure was achieved in equal proportions of cases with or without components separation (Table 2; Fig. 2). PADM was used as a bridge in five cases when fascial closure could not be achieved, three of which involved components separation. Of the five bridged cases, three had a subsequent hernia recurrence as described below.

Previously placed synthetic mesh was removed at the time of surgery in 17 cases. Mean number of drains placed per patient was 3 (median, 4; range, 0-5). Wound VAC (Kinetic Concepts, Inc., San Antonio, TX, USA) was used in 38 of the 45 patient cases: 19 cases with closed incisions, 16 cases with a "French fry" technique, and 3 cases with open wounds; 7 cases did not require VAC. Concurrent surgical procedures performed included panniculectomy $(n=10)$ and stoma takedown $(n=6)$. The mean length of surgery was 192 min (median, 170; range, 58-447).

\section{Postoperative course}

Patients were followed for a mean of 17 months (median, 12; range, 1-48). Thirty-seven of the 45 cases included in 

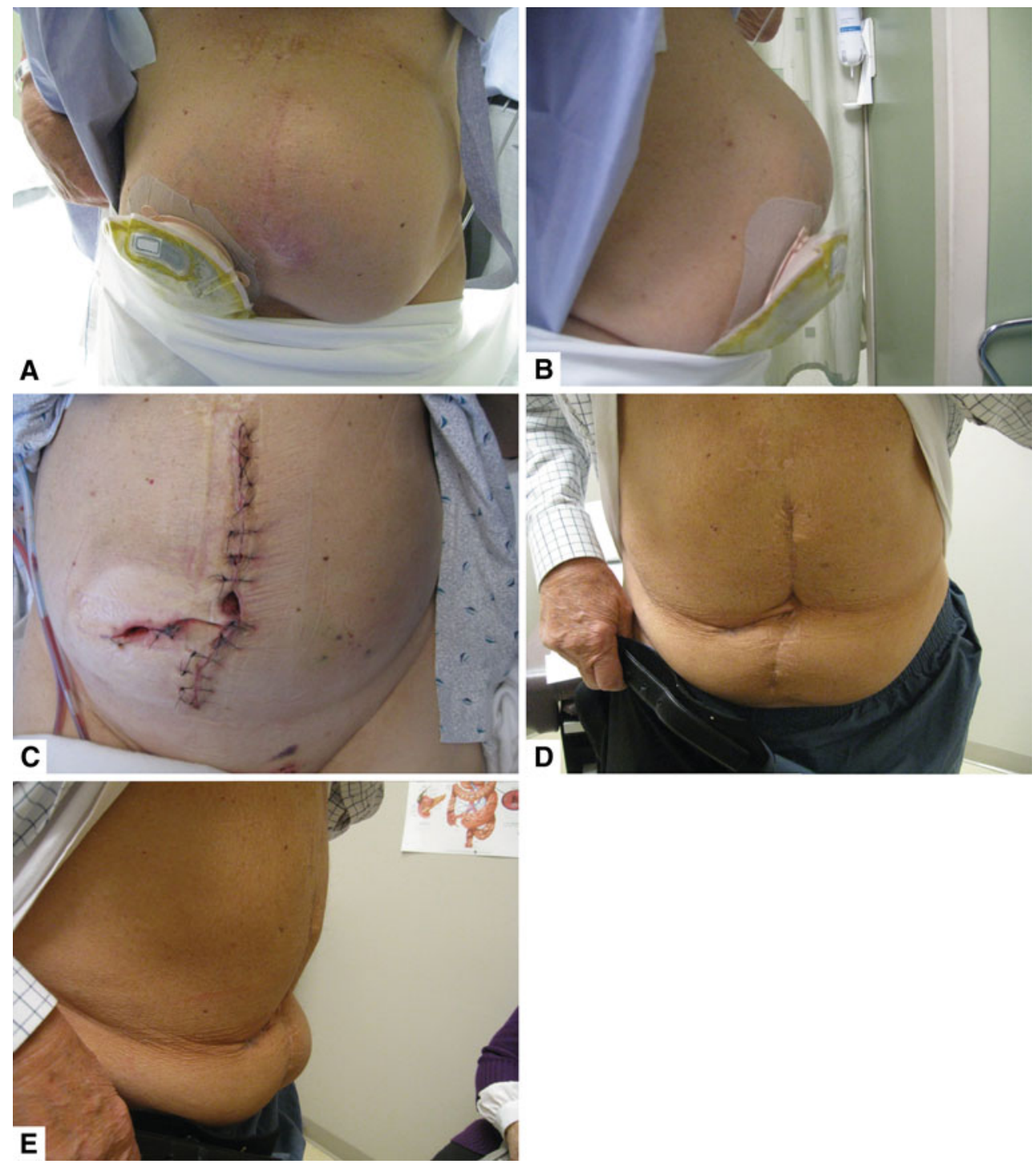

Fig. 3 a, b Large midline incisional hernia and parastomal hernia; c following completion of ileostomy takedown with ileocolic anastomosis, bilateral components separation with repair of incisional

the analysis had clinical follow-up of $\geq 6$ weeks. Of the 25 cases involving patients classified as VHWG Grade 3 risk, 21 had clinical follow-up of $\geq 6$ weeks; all 11 Grade 4 cases had follow-up of $\geq 6$ weeks. A total of 14 cases required postoperative antibiotics for infection, including 11 with preoperative mesh infections and 4 with postoperative deep infections. Antibiotic regimens most often included vancomycin, piperacillin/tazobactam, or ertapenem. Postoperative VAC was applied for a median of 7 days and drains were removed after a median of 2 weeks. The mean length of hospital stay was 8 days (median, 7; range, 3-32). Figure 3 shows a typical preoperative abdominal profile and postoperative results for one of the patients. hernia with PADM, and wound closure using the "French fry" technique; $\mathbf{d}$, e 2 years after surgery

\section{Postoperative assessments}

Postoperative complications were documented. There were $3(6.7 \%)$ skin dehiscences (all treated with local wound care), $4(8.9 \%)$ deep wound infections (all requiring open drainage), and $5(11.1 \%)$ seromas (4 self-limited and 1 requiring drainage). All three events of skin dehiscence occurred in patients with Grade 3 hernias who had successful midline closure achieved with intraperitoneal placement of PADM. Regarding the four patients who experienced deep wound infections, the first patient was a 43 -year-old obese female (BMI, $54 \mathrm{~kg} / \mathrm{m}^{2}$ ), classified as VHWG Grade 3 risk for postsurgical complications, with a history of postoperative wound infections. She underwent a 
panniculectomy and removal of a previously placed Kugel mesh, followed by hernia repair with PADM and VAC using the "French fry" technique. Her wound infection was secondary to a foreign body (black foam fibers left in the wound). There has been no hernia recurrence as of 38 months of follow-up. The second patient with a deep wound infection was a 63-year-old obese female (BMI, $58 \mathrm{~kg} / \mathrm{m}^{2}$ ), classified as VHWG Grade 3 risk, with history of COPD and malnutrition. PADM was placed as a bridge in this patient, with VAC over a closed incision. She developed a wound infection and underwent open drainage in the operating room. The wound healed by secondary intention with a VAC over the exposed PADM. She developed a hernia recurrence at 6 months and underwent repair of her recurrent hernia with synthetic mesh used as a bridge, and had no recurrence through 2 years of follow-up after placement of the synthetic mesh. The third patient with a deep wound infection was a 67-year-old obese male (BMI, $40 \mathrm{~kg} / \mathrm{m}^{2}$ ) classified as VHWG Grade 3 risk. He had a history of postoperative wound infections and underwent concurrent colostomy takedown, diverting loop ileostomy, and midline incisional hernia repair with PADM; VAC was placed on the closed incision. He developed a wound infection due to leakage of enteric ostomy contents into the wound. Open drainage was performed and the wound healed by secondary intention with VAC on the open wound. The PADM was an underlay and was not visually exposed. This patient has had no recurrence at 13 months of follow-up. The fourth patient with a deep wound infection was a 68 -year-old female (BMI, $26 \mathrm{~kg} / \mathrm{m}^{2}$ ) with malnutrition and history of postoperative wound infections. She had an infected Kugel mesh removed and the defect repaired with PADM in a retrorectus fashion. A VAC was placed on the closed incision. She developed a wound infection, which was treated with open drainage. Interestingly, the abscess was in between the PADM and posterior sheath. The PADM was vascularized and completely integrated with the rectus muscle. A drain was placed behind the PADM, the fascia was closed, and VAC was placed on the open wound. She has not experienced a hernia recurrence at 1 year postoperatively. Of the five patients who experienced seromas, four patients had Grade 3 hernias and one had a Grade 4 hernia; two patients had a $\mathrm{BMI}<30 \mathrm{~kg} / \mathrm{m}^{2}$, one had a BMI of $30 \mathrm{~kg} / \mathrm{m}^{2}$, and two had a BMI $>40 \mathrm{~kg} / \mathrm{m}^{2}$. Successful midline closure was achieved in all five of these patients, of whom four had intraperitoneal placement of PADM while one had retrorectus placement of PADM. No seroma patient has experienced a recurrent hernia.

Hernias recurred in 4 of the 45 cases $(8.9 \%)$ overall, and 4 of $37(11.8 \%)$ cases with longer than 6 weeks of follow-up. All patients who did not return for the yearly follow-up underwent a telephone interview and no recurrences were reported. The follow-up time in the current study reflected only those patients who had clinical followup comprising physical examination by the senior author. Hernia recurrences occurred in patients who were at high risk for complications. One recurrence occurred in a Grade 4 obese patient (BMI, $30 \mathrm{~kg} / \mathrm{m}^{2}$ ) who presented with small bowel injury and fecal peritonitis following laparoscopic incisional hernia repair with synthetic mesh. She underwent abdominal washout, repair of the small bowel enterotomy, removal of the fecal-contaminated mesh, and repair of the chronic $20-\mathrm{cm}$ hernia defect with intraperitoneal placement of PADM as a bridge for closure. A VAC was placed using the "French fry" technique after decontamination, and the patient recovered fully within 6 weeks. Unfortunately, she developed a recurrence at 3 months. Two years later, she presented with a symptomatic Grade 3 hernia and underwent bilateral components separation and PADM placement in an underlay fashion with midline rectus muscle closure. She has had no recurrence at 2 years following this operation. The second recurrence occurred in a Grade 2 patient who had a failed mesh repair and multiple comorbidities, including morbid obesity (BMI, $59 \mathrm{~kg} / \mathrm{m}^{2}$ ) and a history of diabetes mellitus, COPD, and current smoking. She underwent removal of failed mesh, repair of the recurrent hernia with PADM in an intraperitoneal position, and a fleur-de-lis panniculectomy. She developed a recurrence 9 months after the initial repair. She was reoperated on for the recurrence and synthetic mesh was placed. She has experienced no recurrence through 1 year of follow-up. Another high-risk patient (BMI, $59 \mathrm{~kg} / \mathrm{m}^{2}$, history of COPD, Grade 3 hernia) had hernia recurrence 6 months following a $15-\mathrm{cm}$ bridged repair with PADM complicated by a colon enterotomy during adhesiolysis. She had a wound infection and the wound healed with VAC over the PADM. Eventually, she underwent surgical repair for the hernia recurrence with synthetic mesh placed as a bridge. The fourth recurrence occurred in a patient who had Grade 4 hernia and was morbidly obese (BMI, $65 \mathrm{~kg} / \mathrm{m}^{2}$ ) with a history of diabetes, COPD, and a large untreated hernia. This patient was in an automobile accident and suffered a lap belt injury with resultant enterocutaneous fistula and full-thickness necrosis of the abdominal wall including the underlying small bowel and colon. She underwent an ileocolectomy and bridged repair with PADM, but within 1 week after surgery she developed an anastomotic leak and recurrent enterocutaneous fistula with loss of the PADM. Her recurrent hernia has been managed without further surgical intervention. Preoperative photographs of the four patients who subsequently had hernia recurrence are shown in Online Resource 1. 


\section{Discussion}

The senior author performed 529 herniorrhaphies with mesh during the study time period. Of those, 235 were ventral/incisional hernias, 219 inguinal, and 75 umbilical. Synthetic mesh was used in all but 45 ventral/incisional hernia repairs. Thus, synthetic mesh also plays a role in this surgical practice, including cases involving Grade 1 and even Grade 2 patients. The senior author is of the opinion that the only absolute indication for biologic matrix use in a VHWG Grade 2 risk patient is immunosuppression. If a patient presents with 3-4 risk factors, the senior author is more likely to use PADM. Synthetic mesh is sometimes used in healthy Grade 3 patients (i.e., previous wound infection) with good results most of the time. Nonetheless, Grade 3 patients with multiple comorbidities, previous wound infection, multiple previous failed synthetic mesh repairs, and/or previous mesh infections should receive a biologic matrix, not a synthetic mesh. Interestingly, of the 45 patients included in this report, 11 (24\%) had infected synthetic mesh removed at the time of surgery and 6 had non-infected synthetic mesh removed at the time of surgery. This represents a total of 17 patients $(38 \%)$ in the population studied who had synthetic mesh removed either for infection or recurrence at the time of surgery, illustrating that synthetic mesh does carry risk of complications. In the subset of 11 patients who had infected mesh removed, a simultaneous single-stage repair was performed with PADM with 2 recurrences at an average 2-year follow-up (12-41 months). Both recurrences occurred following bridged repairs with fecal contamination in patients classified as American Society of Anesthesiologists (ASA) physical status category 4 (life-threatening systemic disease).

This is another demonstration in a growing published experience with the use of non-crosslinked intact PADM for hernia repair. All patients presented with complex ventral abdominal wall hernias, comorbidities, and risk factors for postoperative complications. During a mean of 17 months of postoperative follow-up, surgical outcomes were observed marked by rates of hernia recurrence $(8.9 \%)$, seroma formation (11.1\%), and wound infection $(8.9 \%)$ consistent with prior published reports in similar patients in whom a biologic matrix was used [11-16]. In addition, of the 41 patients with no recurrent hernia, no patient has experienced bulging.

The major challenges posed in the treatment of patients with complex ventral hernias include a history of prior herniorrhaphies (each of which increases the risk of future recurrence) and risk factors that compromise wound healing $[5,6]$. To address some of these challenges, human or animal-derived (porcine or bovine) mesh products have been introduced over the last decade. Large, well- controlled, prospective clinical trials comparing mesh types are lacking and there is currently no consensus regarding the best materials to use in complex abdominal wall hernia patients. Unresolved questions regarding biologic mesh remain, including identification of high-risk patients, optimal surgical techniques, and long-term durability and safety.

Among the available biologic mesh products, PADM has been reported to perform well. In the current investigation, hernia recurrence was seen in four $(8.9 \%)$ cases, all of which involved high-risk patients with multiple comorbidities, including concurrent COPD and morbid obesity in three of the four. This compares favorably to the recurrence rate $(41 \%)$ seen in a similar study of patients who underwent repair using human-derived ADM [17]. The low recurrence rate seen currently is similar to that observed in a prospective, multicenter, observational study of 85 patients undergoing single-stage repair of an infected or contaminated abdominal incisional hernia. In that study, Itani et al. [15] found that PADM facilitated single-stage reconstruction in $80 \%$ of patients; at 12 months, no patients required mesh explantation.

In this study, there were a total of five cases for which PADM was placed as a bridge; three of these patients experienced recurrence within a year. It should be noted that patients in two of these cases had VHWG Grade 4 risk for postsurgical complications. They both had frank fecal contamination at the time of surgery and had grossly infected previously placed mesh. Bridged placement of PADM was chosen as a lifesaving measure. The third bridge failure occurred in a woman with a BMI of $60 \mathrm{~kg} /$ $\mathrm{m}^{2}$ who had a colon enterotomy during lysis of adhesions during her hernia repair. The use of synthetic mesh was contraindicated in all three of these very high-risk cases.

Previously published retrospective cohort studies have also provided support for PADM as an appropriate choice for complex ventral herniorrhaphy [13, 14]. Most recently, Patel et al. [13] conducted a retrospective cohort study in which PADM was used for the primary repair of complex ventral hernias (majority of patients were VHWG Grade 2). No hernia recurrences were observed over a mean followup period of 474 days. The most common complications were related to skin necrosis ( $22 \%)$; other complications, such as seroma $(7.3 \%)$ and wound dehiscence $(7.3 \%)$ were observed. The present set of patients also experienced successful outcomes, despite presenting as higher-risk patients (mean VHWG Grade 3) compared with the patients in the study by Patel et al.

The clinical advantages of biologic mesh may be related to its ability to serve as a biologic scaffold. Unlike synthetic mesh, which tends to become encapsulated with scar tissue, biologic mesh allows revascularization and cellular repopulation, eventually becoming integrated into host 
tissue. Clinically, PADM has been shown to focally integrate well into host tissue with no adverse effects [18]. In one animal study, both human ADM and PADM became infiltrated with host cells and blood vessels within 4 weeks and had good musculofascia-mesh interface strength [19], which may help to prevent hernia recurrence. Importantly, none of the mesh implantations in this animal study showed signs of bowel adhesion formation or infection [19]. Longer-term preclinical studies are needed to describe how biologic mesh strength changes over time as continued tissue remodeling takes place.

The use of a wound VAC in these patients deserves attention. Incisional negative pressure wound therapy (NPWT) following abdominal wall reconstruction and panniculectomy has been shown to significantly improve wound complication rates compared with the use of conventional dressings (22 vs $63 \%$, respectively) [20]. Furthermore, a second study showed similar results in decreasing wound complication rates following abdominal wall reconstruction (18 vs $55 \%$, respectively) [21]. In the current study, 19 patients (15\%) had incisional NPWT with 3 deep wound infections. The "French fry" technique was used in 16 patients, with one $(6 \%)$ postoperative deep wound infection. This technique may prove to be effective in patients with high-risk wounds by reducing tension on the incision line, eliminating fluid from deep wound spaces, obliterating the deep wound space, increasing periwound perfusion, and reducing edema within the skin flaps. Currently in our practice, all patients with VHWG Grade 2 risk have NPWT applied to the closed incision following abdominal wall reconstruction. The "French fry" technique is utilized in Grade 2 patients undergoing concurrent panniculectomy to control the deep wound space in this morbidly obese population. In addition, this technique is preferred in Grade 3 patients who have contamination in the form of enteric contents spillage, intraabdominal abscess, or removal of infected synthetic mesh, as these patients are at high risk for postoperative wound complications and subsequent hernia repair failure.

There is a lack of consensus regarding the most appropriate prosthetic material for patients classified as having VHWG Grade 2 risk; our study included nine patients with Grade 2 risk. The decision to use a biologic matrix for surgical reconstruction in these cases was based on our assessment of each patient's risk profile derived from relevant surgical and medical history in addition to specific VHWG criteria (Table 1) for each individual. Of the nine VHWG Grade 2 patients, all had multiple comorbidities, seven had severe systemic disease (ASA physical classification of 3 or 4), and the other two required removal of a previously placed mesh.

Several limitations of this study deserve mention. The study was retrospective, observational, and did not include a comparative group. The present analysis was not designed to compare clean versus dirty/contaminated groups; outcomes based on such a comparison would be interesting. Although the literature is replete with case reports, larger-scale and longer-term studies evaluating outcomes with PADM versus synthetic mesh are needed to characterize differences regarding postoperative complications (including pain), quality of life, and costs. There is a clear need for additional research to determine costs associated with complex ventral herniorrhaphies. Such costs would presumably be higher than those for lesscomplicated cases (particularly costs associated with length of stay, wound care, and recurrence rates). The postoperative impact on activities of daily living and societal costs (disability, lost productivity, etc.) could also be estimated.

The present study highlights the potential for treating complex ventral hernias in a community hospital setting rather than in a tertiary care center. Despite the fact that many of the patients in this study presented with complex ventral hernias, infection, and comorbidities, nearly all patients had a successful recovery, with only one patient requiring PADM explantation due to factors unrelated to the herniorrhaphy. Several variables are critical to this level of success, including advanced surgical techniques, implantation of a biologic rather than a synthetic mesh, and innovative postoperative wound care. All of these resources can be made available at the community level, making it possible for patients with complex ventral hernias to be treated without the need for referral to a university-based hospital.

\section{Conclusions}

Non-crosslinked intact PADM contributes to favorable early outcomes in complex ventral abdominal wall herniorrhaphy. Long-term studies are needed to elucidate outcomes with PADM relative to synthetic and other biologic mesh products.

Acknowledgments Editorial support for this article was provided by Peloton Advantage, LLC, Parsippany, NJ, and funded by LifeCell, Branchburg, NJ. The opinions expressed in this article are those of the authors. The authors received no honoraria/fee for service or other form of financial support related to the development of this article.

Conflict of interest O. Guerra declares conflict of interest directly related to the submitted work. O. Guerra is a consultant and has received payment for lectures including service on speakers bureaus from LifeCell Corp. M. Macli declares conflict of interest directly related to the submitted work. M. Macli has received honoraria for lectures on AlloDerm ${ }^{\circledR}$ for breast reconstruction surgery from LifeCell Corp. and from KCI for lectures on wound VAC.

Funding This was an Institutional Review Board-approved retrospective cohort study. Editorial support for this article was provided 
by Peloton Advantage, LLC, Parsippany, NJ, and funded by LifeCell Corp., Branchburg, NJ. Dr. Guerra is a consultant for LifeCell Corp. and KCI. Dr. Maclin is a consultant for KCI, LifeCell Corp. and Cook Medical.

Open Access This article is distributed under the terms of the Creative Commons Attribution Noncommercial License which permits any noncommercial use, distribution, and reproduction in any medium, provided the original author(s) and the source are credited.

\section{References}

1. Eid GM, Prince JM, Mattar SG, Hamad G, Ikrammudin S, Schauer PR (2003) Medium-term follow-up confirms the safety and durability of laparoscopic ventral hernia repair with PTFE. Surgery 134:599-603

2. Mudge M, Hughes LE (1985) Incisional hernia: a 10 year prospective study of incidence and attitudes. Br J Surg 72:70-71

3. Israelsson LA, Jonsson T (1996) Incisional hernia after midline laparotomy: a prospective study. Eur J Surg 162:125-129

4. Brenner M, Bochicchio G, Bochicchio K, Ilahi O, Rodriguez E, Henry S, Joshi M, Scalea T (2011) Long-term impact of damage control laparotomy: a prospective study. Arch Surg 146:395-399

5. van 't Riet M, De Vos Van Steenwijk PJ, Bonjer HJ, Steyerberg EW, Jeekel J (2004) Incisional hernia after repair of wound dehiscence: incidence and risk factors. Am Surg 70:281-286

6. Read RC, Yoder G (1989) Recent trends in the management of incisional herniation. Arch Surg 124:485-488

7. Breuing K, Butler CE, Ferzoco S, Franz M, Hultman CS, Kilbridge JF, Rosen M, Silverman RP, Vargo D (2010) Incisional ventral hernias: review of the literature and recommendations regarding the grading and technique of repair. Surgery 148:544-558

8. Luijendijk RW, Hop WC, van den Tol MP, de Lange DC, Braaksma MM, Ijzermans JN, Boelhouwer RU, de Vries BC, Salu MK, Wereldsma JC, Bruijninckx CM, Jeekel J (2000) A comparison of suture repair with mesh repair for incisional hernia. N Engl J Med 343:392-398

9. Franklin ME Jr, Trevino JM, Portillo G, Vela I, Glass JL, Gonzalez JJ (2008) The use of porcine small intestinal submucosa as a prosthetic material for laparoscopic hernia repair in infected and potentially contaminated fields: long-term follow-up. Surg Endosc 22:1941-1946
10. Byrd JF, Jr., Agee N, Getz SB, Lincourt AE, Iannitti DA, Heniford BT (2011) Evaluation of porcine-derived grafts (XenMatrix, Strattice, and Permacol) in an in vivo preclinical study [abstract]. Presented at the Annual Meeting of the American Association of Plastic Surgeons, April 9-12, Boca Raton, FL

11. Adetayo OA, Salcedo SE, Bahjri K, Gupta SC (2011) A metaanalysis of outcomes using acellular dermal matrix in breast and abdominal wall reconstructions: event rates and risk factors predictive of complications. Ann Plast Surg [Epub ahead of print]

12. Butler CE, Campbell KT (2011) Minimally invasive component separation with inlay bioprosthetic mesh (MICSIB) for complex abdominal wall reconstruction. Plast Reconstr Surg 128:698-709

13. Patel KM, Nahabedian MY, Gatti M, Bhanot P (2012) Indications and outcomes following complex abdominal reconstruction with component separation combined with porcine acellular dermal matrix reinforcement. Ann Plast Surg 69:394-398

14. Stromberg J, Zahiri HR, Silverman RP, Singh D (2010) Ventral hernia repair using components separation reinforced with xenogenic Strattice mesh [abstract]. Plast Reconstr Surg 125(suppl 6): 106

15. Itani KMF, Rosen M, Vargo D, Awad SS, DeNoto G, Butler CE, the RICH Study Group (2012) Prospective study of single-stage repair of contaminated hernias using a biologic porcine tissue matrix: the RICH study. Surgery 152:498-505

16. Zhong T, Janis JE, Ahmad J, Hofer SO (2011) Outcomes after abdominal wall reconstruction using acellular dermal matrix: a systematic review. J Plast Reconstr Aesthet Surg 64:1562-1571

17. Maurice SM, Skeete DA (2009) Use of human acellular dermal matrix for abdominal wall reconstructions. Am J Surg 197:35-42

18. Katerinaki E, Zanetto U, Sterne GD (2010) Histological appearance of Strattice tissue matrix used in breast reconstruction. J Plast Reconstr Aesthet Surg 63:e840-e841

19. Campbell KT, Burns NK, Rios CN, Mathur AB, Butler CE (2011) Human versus non-cross-linked porcine acellular dermal matrix used for ventral hernia repair: comparison of in vivo fibrovascular remodeling and mechanical repair strength. Plast Reconstr Surg 127:2321-2332

20. Conde-Green A, Chung TL, Holton L, Hui-Chu H, Zhu Y, Wang H, Zahiri H, Singh DP (2012) Incisional negative-pressure wound therapy versus conventional dressings following abdominal wall reconstruction. Ann Plast Surg [Epub ahead of print]

21. Gassman A, Mehta A, Abtahi A, Bucholz E, Esposito T, Guerra O, Thomas C (2012) Abdominal wall reconstruction: positive outcomes with negative pressure therapy [abstract]. J Am Coll Surg 215:S109 